\begin{tabular}{ll} 
POS & PROCEEDINGS \\
\hline
\end{tabular}

\title{
The Be X-ray Binary Outburst Zoo II
}

\author{
Matthias Kühnel* \\ Remeis-Observatory \& ECAP, Universität Erlangen-Nürnberg, 96049 Bamberg, Germany \\ E-mail: matthias.kuehnel@sternwarte.uni-erlangen.de
}

\section{Peter Kretschmar}

European Space Astronomy Centre (ESA/ESAC), E-28691 Villanueva de la Cañada (Madrid), Spain

\section{Elisa Nespoli}

Observatorio Astronómico de la Univ. de Valencia, C/Catedrático Jose Beltran, 2, 46980

Paterna (Valencia), Spain

European Space Astronomy Centre (ESA/ESAC), E-28691 Villanueva de la Cañada (Madrid), Spain

\section{Atsuo T. Okazaki}

Hokkai-Gakuen University, Toyohira-ku, Sapporo 062-8605, Japan

\section{Gabriele Schönherr}

Leibniz-Institut für Astrophysik Potsdam, An der Sternwarte 16, 14482 Potsdam, Germany

\section{Colleen A. Wilson-Hodge}

NASA Marshall Space Flight Center, Huntsville, AL 35812, USA

\section{Sebastian Falkner}

Remeis-Observatory \& ECAP, Universität Erlangen-Nürnberg, 96049 Bamberg, Germany

\section{Thorsten Brand}

Remeis-Observatory \& ECAP, Universität Erlangen-Nürnberg, 96049 Bamberg, Germany

\section{Friedrich Anders}

Leibniz-Institut für Astrophysik Potsdam, An der Sternwarte 16, 14482 Potsdam, Germany

\section{Fritz-Walter Schwarm}

Remeis-Observatory \& ECAP, Universität Erlangen-Nürnberg, 96049 Bamberg, Germany

\section{Ingo Kreykenbohm}

Remeis-Observatory \& ECAP, Universität Erlangen-Nürnberg, 96049 Bamberg, Germany

\section{Sebastian Müller}

Remeis-Observatory \& ECAP, Universität Erlangen-Nürnberg, 96049 Bamberg, Germany 


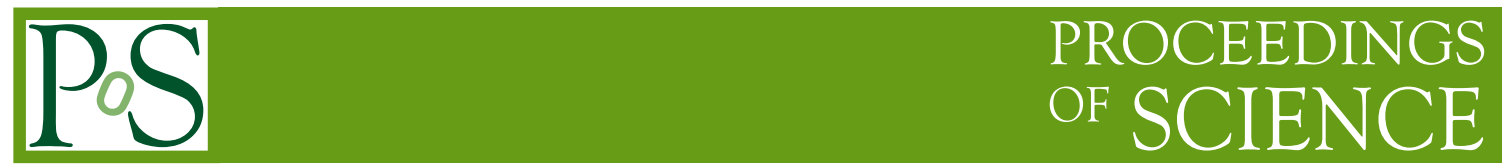

\section{Katja Pottschmidt}

Department of Physics \& Center for Space Science and Technology, UMBC, Baltimore, MD

21250, USA

CRESST \& NASA Goddard Space Flight Center, Greenbelt, MD 20771, USA

Felix Fürst

Cahill Center for Astronomy and Astrophysics, California Institute of Technology, Pasadena, CA 91125, USA

\section{Victoria Grinberg}

Massachusetts Institute of Technology, Kavli Institute for Astrophysics, Cambridge, MA, 02139, USA

\section{Jörn Wilms}

Remeis-Observatory \& ECAP, Universität Erlangen-Nürnberg, 96049 Bamberg, Germany

We have continued our recently started systematic study of Be X-ray binary (BeXRB) outbursts. Specifically, we are developing a catalogue of outbursts including their basic properties based on nearly all available X-ray all-sky-monitors. These properties are derived by fitting asymmetric Gaussians to the outburst lightcurves. This model describes most of the outbursts covered by our preliminary catalogue well; only $13 \%$ of all datasets show more complex outburst shapes. Analyzing the basic properties, we reveal a strong correlation between the outburst length and the reached peak flux. As an example, we discuss possible models describing the observed correlation in $\mathrm{EXO} 2030+375$.

10th INTEGRAL Workshop: "A Synergistic View of the High Energy Sky" - Integral2014, 15-19 September 2014

Annapolis, MD, USA

\footnotetext{
* Speaker.
} 


\section{Be X-ray binary outbursts}

In a Be X-ray binary (BeXRB) a compact object, in all known systems except one ${ }^{1}$ a neutron star, is orbiting a Be-type companion, i.e., an O- or B-type star that shows emission lines in its optical spectrum. These lines originate from a circumstellar disc (Be-disk) around the Be-star. See [16] for a recent review of classical Be-stars. The most prominent emission line, $\mathrm{H} \alpha$, shows strong variability on both short and long timescales. In X-ray binaries, the line profile can evolve from showing one peak to showing two or even more peaks within weeks, while the line flux varies over the years, as found in, e.g., A0535+262 [9]. See [14] for a recent review about BeXRBs.

As opposed to supergiant $\mathrm{O}$ or B-stars, Be stars do not have a strong stellar wind that leads to a constant accretion of matter onto the compact object. Consequently, the binary does not show strong, persistent X-ray emission if the compact object is some $100 \mathrm{lt}-\mathrm{s}$ away from its companion ${ }^{2}$. In case of an eccentric orbit, however, strong X-ray flares may occur around periastron, when the compact object accretes matter from the Be-disk or the companion directly. These type I X-ray outbursts usually last a few weeks, until the source's flux decays below the detection limit of all sky-monitors again. Surprisingly, type I outbursts do not happen every orbital period. In fact, there are only a few systems where regular outbursts have been observed (e.g., GRO J1008-57 [5] or $2 \mathrm{~S} 1845-024$ [3]). The largest fraction of all known systems is in a quiescent state for years to decades until they enter an active state showing a few type I outbursts of different brightnesses (see [11] for a theoretical investigation, and references therein). A BeXRB can also exhibit type II "giant" outbursts that are often not connected to any specific orbital phase, but are almost an order of magnitude brighter than a type I outburst. Intermediate or irregular type outbursts, which show other characteristics, have been observed as well. The unpredictable nature of most BeXRB outbursts, in time as well as in brightness and regarding their evolution, is a current topic of both observational and theoretical work. Discussed scenarios include size changes of the Be star's circumstellar disk (see [1] and references therein), inclined disks with respect to the orbital plane $[12,8]$ ), or precessing and warped Be disks [9]. All these scenarios point at the Be-star as origin for the observed X-ray behaviour. As further evidence, in the case of GRO 1008-57 we have shown that the only varying quantity is likely to be the mass accretion rate [5, 6]. It is defined by the available mass within the system and, thus, by the activity of the Be-type companion star.

In order to investigate the scenarios mentioned above and the connection between the X-ray and the Be-star activities detailed studies of peculiar outbursts as well as of a large sample of similar outbursts across different sources are needed. Only a few of such studies can be found in the literature yet [15]. The main obstacle is a missing outburst catalogue including meta information (basic properties and available observations). The presented work continues the systematic study we have started recently [4] and, especially, starts to close the gap of a missing outburst catalogue.

\footnotetext{
${ }^{1}$ Recently, it has been shown that the B1.5-B2 III star MWC656 is orbited by a 3.8-6.9 $\mathrm{M}_{\odot}$ black hole [2]. Its X-ray luminosity is consistent with a black hole in a quiescent state [10] and currently the black hole does not accrete from its donor star as consistent with current models [13].

${ }^{2}$ There are cases, however, where X-rays are detected during an apparent quiescent state in, e.g., A 0535+26 [17].
} 


\section{A modern catalogue including meta information}

In order to perform an efficient analysis of available all-sky and pointed observations to answer detailed questions about BeXRBs, a complete outburst and activity catalogue of all existing sources is necessary. Furthermore, it is essential to add basic properties about each outburst and the times of available pointed observations to this catalogue. This allows us to perform custom queries based on scientific goals. As basic properties we are in the process of deriving the following aspects for each outburst:

- the date the outburst reached its maximum flux, which we use as identifier

- lightcurve properties such as the duration of the rise and decline and the maximum flux

- the outburst shape and the occurrence of short flares or peculiarities

- simple spectral information like the hardness ratio

- binary information, for instance the orbital phase

In order to investigate the spectral information and to constrain the other properties in the best way, we use the following instruments and energy ranges:

- Integral-ISGRI ${ }^{3}(17-80 \mathrm{keV})$ and $-\mathrm{JEM}^{\mathrm{X}} \mathrm{X}^{3}(3-35 \mathrm{keV})$

- Swift-BAT ${ }^{4}(15-50 \mathrm{keV})$

- $C G R O-$ BATSE $^{5}(20-160 \mathrm{keV})$

- $R X T E-\mathrm{ASM}^{6}(2-10 \mathrm{keV})$

- $\operatorname{MAXI}^{7}(3-20 \mathrm{keV})$

The lightcurve properties can be obtained by fitting the observed outburst with a model describing its general shape. Since we are mainly interested in parameters like the maximum flux, at which date it is reached, and the rise and decline time, the model will not take into account pre- or postflares or flux variations on the order of a few days.

A few observed outbursts seem to rise or fall linearly, for example the decline of the 2008 March/April outburst of 4U 0115+634 in hard X-rays (see the BAT-lightcurve as shown in Fig. 3). A model describing the outburst linearly does not, however, work for the majority of sources and outbursts. Furthermore, residuals remain in some cases, which requires a sudden slope change of the linear model. We find that an asymmetric Gaussian with an optional plateau at the maximum is able to describe most of the observed outbursts and does not require a slope change. Its flux

\footnotetext{
${ }^{3}$ HEAVENS lightcurves: http://isdc.unige.ch/heavens/

${ }^{4}$ BAT Hard X-ray Survey: http://swift.gsfc.nasa.gov/results/bs70mon/

${ }^{5}$ BATSE Earth Occultation lightcurves: http://www.batse.msfc.nasa.gov/batse/occultation/list.html

${ }^{6}$ ASM lightcurves: http://heasarc.gsfc.nasa.gov/docs/xte/ASM/sources.html

${ }^{7}$ MAXI lightcurves: http://maxi.riken.jp/mxondem/
} 

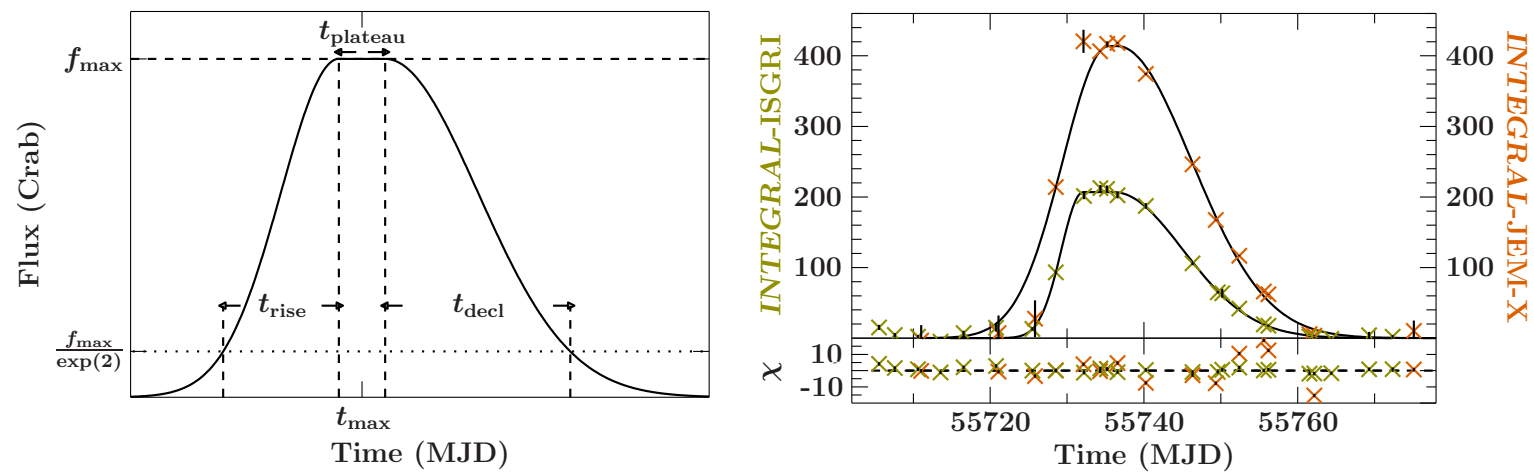

Figure 1: The asymmetric Gaussian model (left) as defined in Eq. 2.1 is applied to the Integral-ISGRI and -JEM-X lightcurves of the 2011 June outburst of 4U 0115+634 (right). Fluxes are in mCrab in the instrument's energy range and error bars are included. The model provides a good overall description of the outburst evolution. However, the residuals cause a high $\chi_{\text {red }}^{2} \approx 65$ due to small flux changes on timescales of a day, which are detected because of the good quality of the Integral-data.

evolution $f(t)$ over an outburst is defined as:

$$
f(t)= \begin{cases}f_{\max } \exp \left(-2\left(\frac{t-t_{\text {max }}+0.5 t_{\text {plateau }}}{t_{\text {rise }}}\right)^{2}\right) & , t<t_{\text {max }}-0.5 t_{\text {plateau }} \\ f_{\max } & ,-0.5 t_{\text {plateau }} \leq t-t_{\text {max }} \leq 0.5 t_{\text {plateau }} \\ f_{\text {max }} \exp \left(-2\left(\frac{t-t_{\text {max }}-0.5 t_{\text {plateau }}}{t_{\text {decl }}}\right)^{2}\right) & , t>t_{\text {max }}+0.5 t_{\text {plateau }}\end{cases}
$$

with the maximum flux, $f_{\max }$, during a plateau with the duration, $t_{\text {plateau }}$, and its mid-time, $t_{\max }$. The rise- and decline-time, $t_{\text {rise }}$ and $t_{\text {decl }}$, respectively, represent the $2 \sigma$ interval of the corresponding Gaussian. Using this choice of parameters we define the outburst length, $t_{\text {length }}$, to be

$$
t_{\text {length }}=t_{\text {rise }}+t_{\text {decl }}+t_{\text {plateau }}
$$

During this time more than $95 \%$ of the integrated flux is covered. If we assume that the mass accretion rate is proportional to the observed flux, it means that $95 \%$ of the total mass is accreted during $t_{\text {length }}$.

The asymmetric Gaussian model as defined in Eq. 2.1 is illustrated in Fig. 1 (left). As an example the individual fits to the Integral-ISGRI and -JEM-X lightcurves to the 2011 June outburst of 4U 0115+634 are shown as well (right). Although the general flux evolution over the $\sim 40$ days of activity is described successfully, the fit quality of $\chi_{\text {ref }}^{2} \approx 65$ with 34 d.o.f. is not indicating a good fit. The reason is the good quality of the Integral flux measurements, resulting in small uncertainties. These reveal a short term flux variation on the order of a few days, especially in JEM-X at lower X-ray energies, which are not described by our simple model. A model describing such short-term variations is, however, outside of the scope of our work. The asymmetric Gaussian model is sufficient to reach our goal to determine basic outburst parameters for our catalogue.

\subsection{Preliminary outburst catalogue}

So far, our preliminary catalogue of BeXRBs contains 16 sources (see Table 1). After the lightcurves of the different instruments listed above were rebinned to a $1 \mathrm{~d}$ time resolution we 


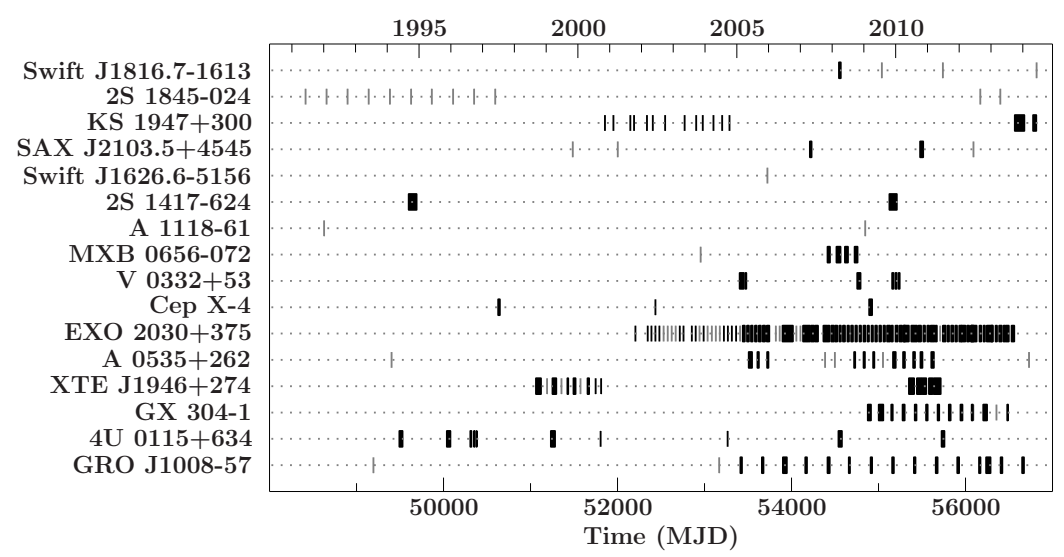

Figure 2: The activity periods for the 16 sources in our preliminary catalogue as detected in all used instruments. The duration of each activity period, i.e., the thickness of the lines, corresponds to the outburst length (see Eq. 2.2) after a fit to the model defined in Eq. 2.1. Lines drawn in gray represent outbursts with a low signal quality or where the model is unable to describe the outburst flux evolution.

have added almost all outbursts and activity periods that can be detected by eye to the catalogue. This leads to 217 detected outbursts (see Table 1 and Fig. 2) and 351 individual datasets (there is one dataset for each outburst and detector). We are in the process of systematically analyzing the lightcurves using the Bayesian Block detection technique as developed by [18] to add weaker outbursts to the catalogue. Furthmore, we will include activity periods as reported in the literature.

After having identified an outburst of a source in any of the instruments we have fitted the asymmetric Gaussian model as defined in Eq. 2.1 to the corresponding lightcurves. The time range for the fit is selected starting at the maximum observed flux until the data of three consecutive days have a signal-to-noise ratio smaller than two. Detected pre- or post-flares are ignored. The fit-strategy includes a first fit without a plateau to get reasonable start parameters for a secondary fit, where a plateau is allowed. This prevents weak outbursts from being fitted by a plateau alone, i.e., with a negligible rise- or decline-time. If the plateau length is consistent with zero at the three sigma level it is fixed to zero.

From all 315 datasets in our preliminary catalogue, $63 \%$ could be fitted successfully with the asymmetric Gaussian model. $67 \%$ of the failed fits, i.e., $25 \%$ of all datasets, are due to a low signal-to-noise ratio. The remaining 42 datasets show complex outburst shapes where the general flux evolution does not follow an asymmetric Gaussian.

Figures 3 and 4 show examples of successful fits to the data of both the soft (RXTE-ASM or MAXI) and hard X-ray lightcurves (Swift-BAT). In the case of the $2008 \mathrm{March} / \mathrm{April}$ outburst of $4 \mathrm{U} 0115+634$ (Fig. 3), differences in the declining phase between the soft and hard X-rays are revealed. In contrast to $4 \mathrm{U} 0115+634$, the peak of the 2010 July outburst of EXO 2030+375 in the soft X-rays seems to be delayed compared to its hard X-ray lightcurve (see Fig. 4). Unfortunately, the decline of the outburst was not covered by MAXI.

As an example for complex outbursts included in our catalogue Figures 5 and 6 show the 2003 October/November outburst of MXB 0656-072 as observed by RXTE-ASM and the 2013 March activity of GX 304-1 as seen by Swift-BAT, respectively. In the first strong daily flaring with an amplitude around a factor of two is seen after a short rising part. After around 40 days the 
Table 1: The sources in our preliminary catalogue and detected number of outbursts. The coordinates (RA \& DEC) are from Simbad $^{\mathrm{a}}$.

\begin{tabular}{lllc}
\hline \hline Source & RA & DEC & Outbursts \\
\hline 4U 0115+634 & $01 \mathrm{~h} 18 \mathrm{~m} 31.90 \mathrm{~s}$ & $+63^{\circ} 44 \mathrm{~m} 24.0 \mathrm{~s}$ & 10 \\
V 0332+53 & 03h34m59.905s & $+53^{\circ} 10 \mathrm{~m} 23.34 \mathrm{~s}$ & 7 \\
1A 0535+262 & 05h38m54.573s & $+26^{\circ} 18 \mathrm{~h} 56.83 \mathrm{~s}$ & 16 \\
MXB 0656-072 & 06h58m17.29s & $-07^{\circ} 12 \mathrm{~h} 35.2 \mathrm{~s}$ & 5 \\
GRO J1008-57 & $10 \mathrm{~h} 09 \mathrm{~m} 44 \mathrm{~s}$ & $-58^{\circ} 17.7 \mathrm{~m}$ & 17 \\
1A 1118-61 & $11 \mathrm{~h} 20 \mathrm{~m} 57.177 \mathrm{~s}$ & $-61^{\circ} 55 \mathrm{~m} 00.24 \mathrm{~s}$ & 2 \\
GX 304-1 & $13 \mathrm{~h} 01 \mathrm{~m} 17.10 \mathrm{~s}$ & $-61^{\circ} 36 \mathrm{~h} 06.6 \mathrm{~s}$ & 15 \\
2S 1417-624 & $14 \mathrm{~h} 21 \mathrm{~m} 12.8 \mathrm{~s}$ & $-62^{\circ} 41 \mathrm{~m} 54 \mathrm{~s}$ & 2 \\
Swift J1626.6-5156 & $16 \mathrm{~h} 26 \mathrm{~m} 36.24 \mathrm{~s}$ & $-51^{\circ} 56 \mathrm{~m} 33.5 \mathrm{~s}$ & 1 \\
Swift J1816.7-1613 & $18 \mathrm{~h} 16 \mathrm{~m} 42.66 \mathrm{~s}$ & $-16^{\circ} 13 \mathrm{~m} 23.4 \mathrm{~s}$ & 4 \\
2S 1845-024 & $18 \mathrm{~h} 48 \mathrm{~m} 17.7 \mathrm{~s}$ & $-02^{\circ} 25 \mathrm{~h} 13 \mathrm{~s}$ & 12 \\
XTE J1946+274 & $19 \mathrm{~h} 45 \mathrm{~m} 39.36 \mathrm{~s}$ & $+27^{\circ} 21 \mathrm{~h} 55.5 \mathrm{~s}$ & 15 \\
KS 1947+300 & $19 \mathrm{~h} 49 \mathrm{~m} 35.49 \mathrm{~s}$ & $+30^{\circ} 12 \mathrm{~h} 31.8 \mathrm{~s}$ & 15 \\
EXO 2030+375 & $20 \mathrm{~h} 32 \mathrm{~m} 15.28 \mathrm{~s}$ & $+37^{\circ} 38 \mathrm{~h} 14.9 \mathrm{~s}$ & 88 \\
SAX J2103.5+4545 & $21 \mathrm{~h} 03 \mathrm{~m} 35.71 \mathrm{~s}$ & $+45^{\circ} 45 \mathrm{~h} 05.5 \mathrm{~s}$ & 5 \\
Cep X-4 & $21 \mathrm{~h} 39 \mathrm{~m} 30.684 \mathrm{~s}$ & $+56^{\circ} 59 \mathrm{~m} 10.45 \mathrm{~s}$ & 3 \\
\hline \hline
\end{tabular}

${ }^{a}$ http://simbad.u-strasbg.fr/simbad/

flaring stops and the outburst decays smoothly into quiescence. In the latter source three outbursts of $\sim 10$ days duration are detected, which partly overlap. Outside of this unusual activity residual emission is detected. The reason for the interesting behavior of these two examples is not understood yet [need to check]. Such peculiar outbursts will be marked in our catalogue to easily identify peculiarities and allow for both detailed studies and quantitative comparisons.

\subsection{Examples of meta data analysis}

Once we have fitted the asymmetric Gaussian model of Eq. 2.1 to the many outbursts of a particular source we can start to investigate the resulting fit parameters quantitatively. As a prime example Fig. 7 (left) shows the dependency of the outburst length of EXO 2030+375 as defined in Eq. 2.2 on the peak luminosity reached during each outburst. A clear correlation is visible: the brighter an outburst the longer it lasts. The observed flux has been converted into units of the Crab and then into luminosity relative to that of the Crab by $d^{2} / d_{\text {crab }}^{2}$, where $d=7.1(2) \mathrm{kpc}$ is the distance to EXO 2030+375 [19] and $d_{\text {crab }}=2.0 \mathrm{kpc}$ the assumed distance to the Crab [7]. The correlation between the outburst length and the peak flux is confirmed by all instruments used and roughly follows a power-law. A simple fit of the correlation, however, results in a bad fit (3; gray, dashed line) owing to residuals below a luminosity of $\sim 1$ Crab. A minimum non-zero outburst length, i.e., a constant offset of the power-law results in a better description of the correlation (2; black, dashed line). Further investigation reveals, however, that the discrepancy to a simple power-law is mainly driven by the observed type II "giant" outburst in 2006 August that reached a luminosity 


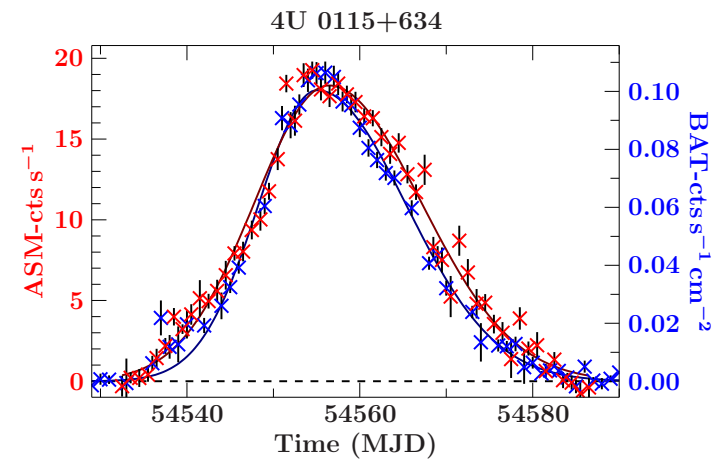

Figure 3: The 2008 March/April outburst of 4U 0115+634 as seen with RXTE-ASM and SwiftBAT. Although differences are visible in both lightcurves, the asymmetric Gaussian model is able to fit the data.

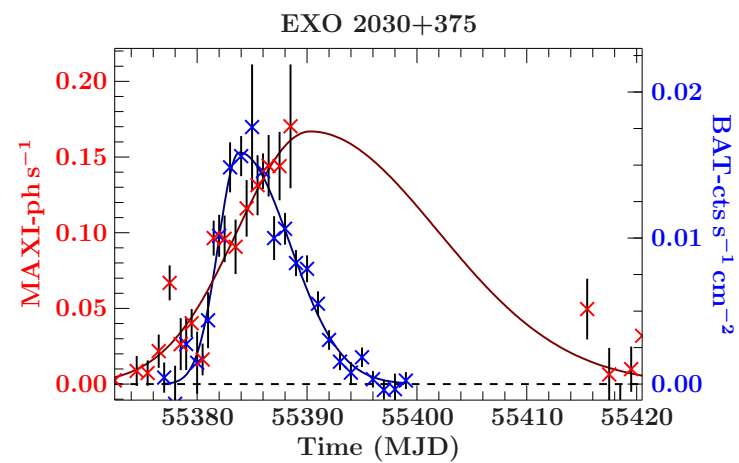

Figure 4: Data and fitted model to the 2010 July outburst of EXO 2030+375. The peak in the soft $\mathrm{X}$-rays as measured by MAXI seems to be delayed relative to the hard X-rays in Swift-BAT.

around 10 Crab in Swift-BAT and lasted for more than 100 days. EXO 2030+375 exhibits one outburst during each orbital period, which is $P_{\text {orb }}=46.02 \mathrm{~d}$ [20]. Thus, the "giant" outburst spans more than two full orbits as opposed to all other observed outbursts of the source. In fact, this outburst might also be an overlap of two strong type I outbursts, which may explain the complex outburst shape as shown in Fig. 7 (right). If we exclude the parameters of the "giant" outburst from the correlation, a simple power-law describes the data (1; solid, black line) as well as a power-law with a constant offset. There is evidence that the other 15 sources in our catalogue show similar correlations between the peak luminosity and the outburst length. We are currently investigating the parameters of the correlations among all sources in our sample.

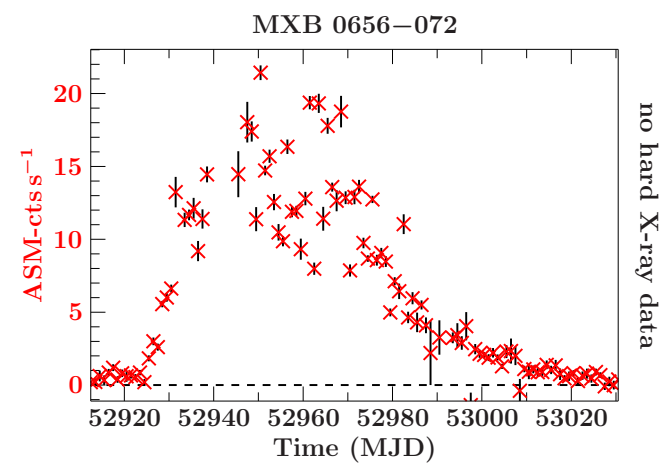

Figure 5: An extreme flaring period of about 40 days in duration is detected in RXTE-ASM after the rise of the 2003 October/November outburst of MXB 0656-072.

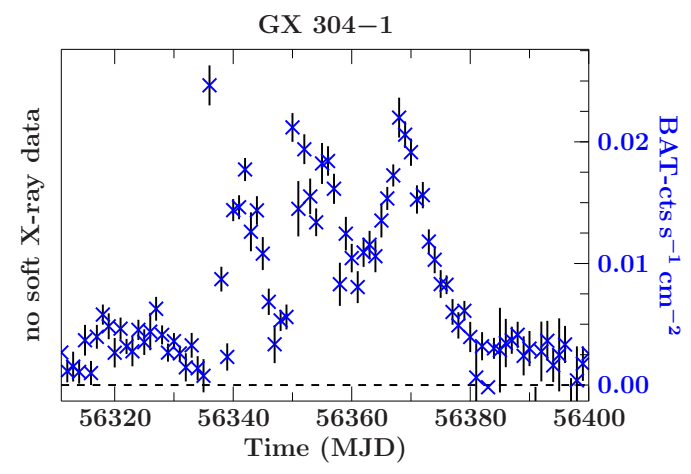

Figure 6: Three weak, overlapping outbursts of GX 304-1 in 2013 March. Apart from some residual emission, the total activity period is not longer than 40 days. 

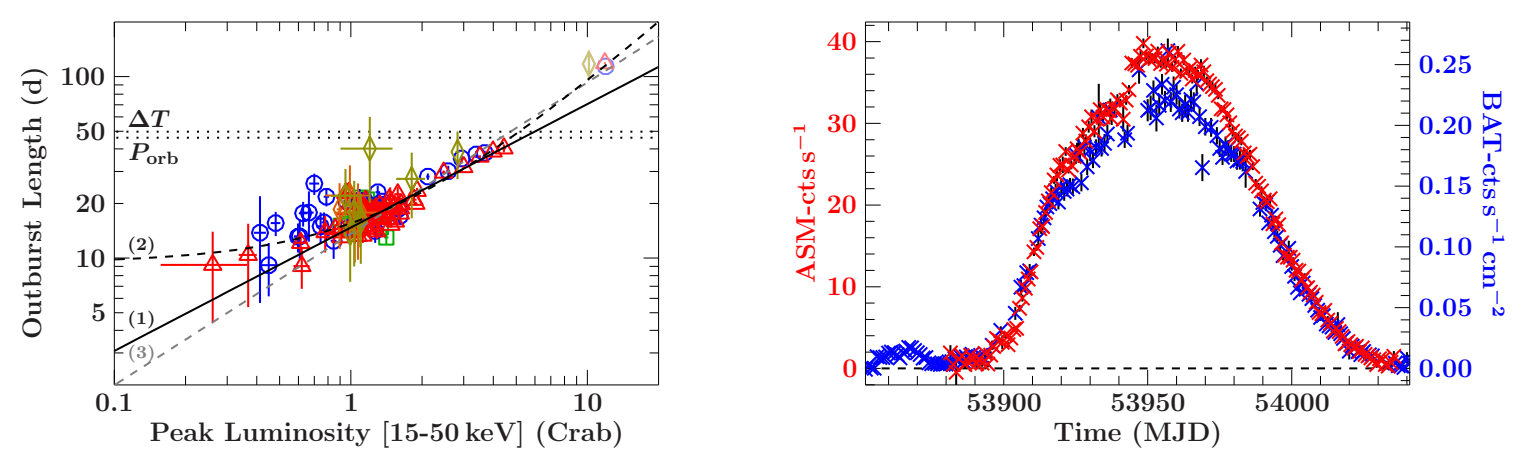

Figure 7: The fitted length of each outburst of EXO 2030+375 detected in Swift-BAT (red), RXTE-ASM (blue), MAXI (green), Integral-ISGRI (yellow) and Integral-JEM-X (orange) over the outburst's peak luminosity (left). The luminosities have been shifted to match the $S$ wift-BAT measurements. A clear correlation is visible, which can be described by a power-law with a constant offset (2: black, dashed line). A fit without the offset (3; gray, dashed line) results in a worse description. The type II outburst with a luminosity around 10 Crab with a duration of more than 100 days (lightcurve on the right) is longer than both the orbital period $P_{\text {orb }}$ (indicated by the lower dotted line in the left plot) and the usual time span $\Delta T$ (upper dotted line) between two consecutive outbursts. If this outburst is ignored in the correlation (left; transparent points), a simple power-law (1; black, solid line) results in a statistically equal description to the power-law with a constant offset for all data(2).

\section{Outlook}

The list of outbursts in our preliminary catalogue presented here is currently being extended to include weaker outbursts and activity periods as reported in the literature. The final catalogue will be published soon (Kretschmar et al., in prep.) and will be kept up-to-date in terms of recent outburst and sources.

The analysis of the outburst properties, especially the correlation between the peak luminosity and the outburst length, is finalized. We will use recent theoretical work by [12] to calculate the expected outburst shapes based on simulated mass accretion rates. The resulting shapes will be compared and fitted to the outbursts in our catalogue (Kühnel et al., in prep.).

In addition to the catalogue, we are also investigating individual sources. As shown in Fig. 7 (left) the mean time span $\Delta T=49.8(1.7) \mathrm{d}$ between two consecutive outbursts of EXO 2030+375 is slightly larger than its orbital period of $P_{\text {orb }}=46.02 \mathrm{~d}$. Although the reason for this $2.2 \sigma$ observation might be of statistical nature, further analysis of meta information shows a significant change of the orbital phase, at which the peak flux is reached, over time (Wilson-Hodge et al., in prep.).

\section{Acknowledgments}

M. Kühnel acknowledges funding by the Bundesministerium für Wirtschaft und Technologie under Deutsches Zentrum für Luft- und Raumfahrt grant 50OR1113. The figures shown in this paper were created using the SLXfig module, which was developed by John E. Davis. We thank the organizers of the 10th Integral Workshop "A Synergistic View of the High Energy Sky" for the the useful discussions triggered by this interesting and well organized workshop. 


\section{References}

[1] Camero-Arranz, A., Finger, M. H., Wilson-Hodge, C. A., et al., X-Ray and Optical Observations of A 0535+26, ApJ 754, A20 (2012)

[2] Casares, J., Negueruela, I., Ribó, M., et al., A Be-type star with a black-hole companion, Nature 505, Issue 7483, 378-381 (2014)

[3] Finger, M. H., Bildsten, L., Chakrabarty, D., et al., The Outbursts and Orbit of the Accreting Pulsar GS 1843-02 = 2S 1845-024, ApJ 517, 449-459 (1999)

[4] Kretschmar, P., Nespoli, E., Reig, P., Anders, F., The Be X-ray Binary Outburst Zoo, 2012, in: Conf. Proc. of the 9th Integral Workshop, Proceedings of Science, article 16 (2012)

[5] Kühnel, M., Müller, S., Kreykenbohm, I., et al., GRO J1008-57: an (almost) predictable transient $X$-ray binary, A\&A 555, A95 (2013)

[6] Kühnel, M., Müller, S., Kreykenbohm, I., et al., Luminosity dependent accretion state change in GRO J1008-57, in: Physics at the Magnetospheric Boundary, EPJ Web of Conf. 64, article 06003 (2014)

[7] Manchester, R. N., Hobbs, G. B., Teoh, A., Hobbs, M., The Australia Telescope National Facility Pulsar Catalogue, AJ 129, Issue 4, 1993-2006 (2005)

[8] Martin, R, G., Nixon, C., Armitage, P. J., et al., Giant Outbursts in Be/X-Ray Binaries, ApJ 790, L34 (2014)

[9] Moritani, Y., Nogami, D., Okazaki, A. T., et al., Precessing Warped Be Disk Triggering the Giant Outbursts in 2009 and 2011 in A0535+262/V725Tau, PASJ 65, A83 (2013)

[10] Munar-Adrover, P., Paredes, J. M., Ribó, M., et al., Discovery of X-Ray Emission from the First Be/Black Hole System, ApJ 786, Issue 2, L11 (2014)

[11] Okazaki, A. T., Negueruela, I., A natural explanation for periodic X-ray outbursts in Be/X-ray binaries, $A \& A$ 377, 161-174 (2001)

[12] Okazaki, A. T., Hayasaki, K., Moritani, Y., Origin of Two Types of X-Ray Outbursts in Be/X-Ray Binaries. I. Accretion Scenarios, PASJ 65, A41 (2013)

[13] Plotkin, R. M., Gallo, E., Jonker, P. G., The X-Ray Spectral Evolution of Galactic Black Hole X-Ray Binaries toward Quiescence, ApJ 773, A59

[14] Reig, P., Be/X-ray binaries, Ap\&SS 332, 1-29 (2011)

[15] Reig, P., Nespoli, E., Patterns of variability in Be/X-ray pulsars during giant outbursts, A\&A 551, A1 (2013)

[16] Rivinius, T., Carciofi, A. C, Martayan, C., Classical Be stars. Rapidly rotating B stars with viscous Keplerian decretion disks, A\&ARv 21, A69 (2013)

[17] Rothschild, R., Markowitz, A., Hemphill, P., et al., Observations of the High-mass X-Ray Binary A 0535+26 in Quiescence, ApJ 770, A19 (2013)

[18] Scargle, J. D., Norris, J. P., Jackson, B., et al., Studies in Astronomical Time Series Analysis. VI. Bayesian Block Representations, ApJ 764, A167 (2013)

[19] Wilson, C. A., Finger, M. H., Coe, M. J., et al., A Decade in the Life of EXO 2030+375: A Multiwavelength Study of an Accreting X-Ray Pulsar, ApJ 570, 287-302 (2002)

[20] Wilson, C. A., Finger, M. H., Camero-Arranz, A., Outbursts Large and Small from EXO 2030+375, ApJ 678, Issue 2, 1263-1272 (2008) 